\title{
Preisbildung im genossenschaftlichen System am Beispiel der Milchwirtschaft
}

\author{
Nina Steffen \\ Stephanie Schlecht \\ Achim Spiller*
}

\section{Zusammenfassung}

Der Beitrag greift die Preisbildung in Genossenschaften des Agribusiness am Beispiel der Milchwirtschaft auf. Mit Hilfe einer empirischen Erhebung bei Landwirten werden verschiedene Einflussfaktoren auf die Bewertung des Preissystems identifiziert. Es zeigt sich eine deutliche Ablehnung der klassischen genossenschaftlichen Preisfindung, in der die Landwirte als Anteilseigner über die Gremien Einfluss auf den Auszahlungspreis nehmen können.

\section{Summary}

This article discusses the price formation in agricultural markets using the price determination in the cooperative dairy industry as an example. Contrary to traditional hypotheses, an empirical investigation shows that the members of dairy cooperatives do not have direct control over payout-prices.

\section{Résumé}

Cet article traite de la formation des prix dans les coopératives de «l'agrobusiness » à partir de l'exemple des industries coopératives laitières. Cette étude empirique permet d'identifier plusieurs facteurs influant sur la formation des prix. Il en ressort que, contrairement aux mécanismes coopératifs classiques, les membres des coopératives laitières n'ont pas de contrôle direct sur les prix.

\section{Einleitung}

Im Agribusiness sind Genossenschaften, in denen die Lieferanten gleichzeitig Anteilseigner sind, von besonderer Bedeutung. ${ }^{1}$ Diese genossenschaftlichen Unternehmen, im internationalen Schrifttum als „Marketing Cooperatives“ bezeichnet, ver-

Prof. Dr. Achim Spiller, Nina Steffen (M. Sc.) und Stephanie Schlecht (M. Sc.) sind für den Arbeitsbereich Marketing für Lebensmittel und Agrarprodukte des Departments für Agrarökonomie und Rurale Entwicklung an der Georg-August-Universität Göttingen zuständig.

1 Vgl. Gerlach, S./Köhler, B./Spiller, A.: Landwirte als Lieferanten und Unternehmenseigner: Zum Management von Supplier- und Investor-Relations im Agribusiness, in: 
arbeiten und vermarkten die Rohwaren der Lieferanten mit dem Ziel, die besten Konditionen für ihre Mitglieder zu erwirtschaften. ${ }^{2}$ In Deutschland ist diese Unternehmensform in den Bereichen der Milch-, Fleisch-, Zucker-, Wein- sowie Obst- und Gemüsewirtschaft marktführend.

Insbesondere bei den milchverarbeitenden Genossenschaften, die rund zwei Drittel der in Deutschland erzeugten Milch vermarkten, ${ }^{3}$ weisen der Milchstreik aus dem Jahr 2008 und die anhaltenden Proteste auf eine Unzufriedenheit der Landwirte mit ihren Abnehmern hin. Neben der Höhe der Auszahlungspreise steht auch die Form der Preisfestlegung in Genossenschaftsmolkereien in der Kritik. Des Weiteren werden die Rahmenbedingungen der europäischen Milchwirtschaft mittelfristig mit der Quotenabschaffung im Wirtschaftsjahr 2014/2015 gravierend verändert. ${ }^{4}$ Der Übergang von einem durch das staatliche Quotensystem regulierten in einen freien Markt stellt die Genossenschaftsmolkereien und ihr traditionelles Preissystem vor eine große Herausforderung. Vor diesem Hintergrund zielt die vorliegende Arbeit darauf ab, das aktuelle System der genossenschaftlichen Preisbildung aus Sicht der Lieferanten zu analysieren und Einflussfaktoren auf die Bewertung zu identifizieren. Die Studie liefert damit einen Beitrag zum Lieferantenmanagement von genossenschaftlichen Unternehmen.

\section{Möglichkeiten der Preisfindung in der Milchwirtschaft}

Das „Milchgeld“, d. h. die Rohstoffvergütung der Molkerei an die Landwirte, ist für spezialisierte landwirtschaftliche Milchlieferanten die wichtigste Einkommensquelle. Daneben existieren nur geringe Einnahmen aus Kälber- und Schlachtviehverkäufen sowie die Direktzahlungen der Europäischen Union. ${ }^{5}$ Für die Existenzsicherung der landwirtschaftlichen Betriebe ist demzufolge ein kos-

Darnhofer, I./Pöchtrager, S./Schmid, E. (Hrsg.): Jahrbuch der österreichischen Gesellschaft für Agrarökonomie Bd. 14 (2005), Wien, S. 222-224.

2 Vgl. Klose, H.: Zusatzgrundkapital für eingetragene Genossenschaften: neue Wege zur Stärkung der Eigenkapitalausstattung eingetragener Genossenschaften, Marburger Schriften zum Genossenschaftswesen Bd. 90 (1998), Göttingen, S. 32 f.

3 Vgl. Deutscher Raiffeisenverband e. V.: Milchwirtschaft, URL:

http://www.raiffeisen.de/genossenschaften/sparten/milch.htm, Abrufdatum: 24.02.2009.

${ }_{4}$ Vgl. Fahlbusch, M./Bahr, A./Brümmer, B./Spiller, A.: Der Markt für Milch und Milcherzeugnisse, in: Agrarwirtschaft 1/2009, S. 36; EU-Kommission: Vorbereitung auf den „GAP-Gesundheitscheck“, Mitteilung der Kommission an den Rat und das Europäische Parlament, Brüssel 2007, S. 8.

5 Vgl. Wocken, C./Spiller, A.: Der Einfluss des Preises auf die Stabilität von Geschäftsbeziehungen in der Agrar- und Ernährungswirtschaft, in: Wocken, C.: Management von Geschäftsbeziehungen in der Milchwirtschaft, Göttingen 2008, S. 205; Weindlmaier, H./Huber, A.: Vor- und Nachteile des bayerischen Erzeugerorientierungspreises (EOP), in: Deutsche Milchwirtschaft 25/2001, S. 1087. 
tendeckender Milchpreis ebenso wie eine sichere Abnahme der produzierten Milch unerlässlich. Es können Zielkonflikte entstehen, da die sichere Abnahme der Milch nur von einer wirtschaftlich stabilen Molkerei langfristig gewährleistet werden kann. Dies führt gegebenenfalls zu niedrigeren Auszahlungspreisen. Die Preisfindung zwischen Molkereien und ihren Lieferanten kann durch drei verschiedene Systeme erfolgen (vgl. Abbildung $1^{6}$ ). Neben dem traditionellen Genossenschaftssystem (vgl. Kapitel II a) sind vor allem zwei weitere Preissysteme von Bedeutung: Entweder kann der Preis auf Basis eines Referenzpreises mit Auf- und Abschlägen festgelegt werden oder der Preis wird direkt zwischen den Milcherzeugern bzw. Milcherzeugergemeinschaften und der Molkerei ausgehandelt.

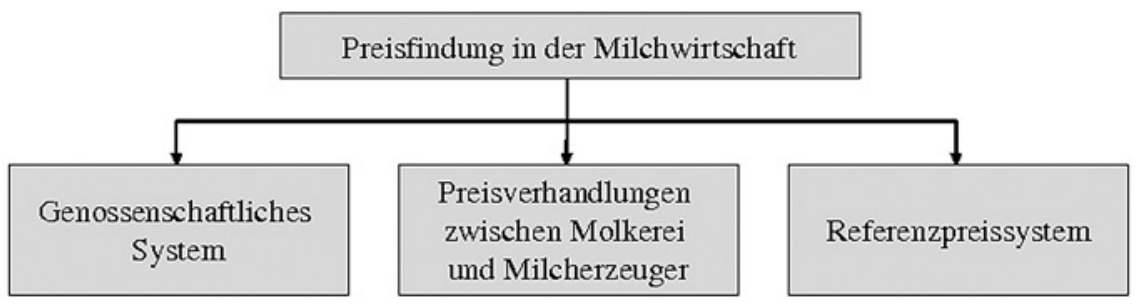

$A b b$. 1: Preisfindungssysteme in der deutschen Milchwirtschaft

\section{a) Genossenschaftliches System}

Die Preisfestsetzung einer Genossenschaftsmolkerei erfolgt nach dem tatsächlichen Verwertungsergebnis. ${ }^{7}$ Dabei ist die notwendige Orientierungsgröße die so genannte Nettoverwertung (des Vorvormonats). Diese ist der Verkaufserlös der Produkte (=Bruttoverwertung) abzüglich der Kosten für Erfassung, Herstellung und Vermarktung. ${ }^{8}$ Die Preisfestlegung erfolgt mithin rückwirkend und einseitig durch den Vorstand „,nach pflichtgemäßem Ermessen“" . Der Vorstand wird von den Mitgliedern gewählt, so dass für die Milchlandwirte die Möglichkeit besteht, hierauf Einfluss zu nehmen. Der festgelegte Milchauszahlungspreis darf nach der Beschlussfassung nicht

\footnotetext{
6 Eigene Darstellung.

7 Vgl. Weindlmaier, H.: Absatz- und Beschaffungsmarketing als Rahmenbedingungen für die Wettbewerbsfähigkeit des Molkereisektors in Deutschland, in: Anhang 2 zum Gutachten des Wissenschaftlichen Beirats beim Bundesministerium für Ernährung, Landwirtschaft und Forsten: Zur Wettbewerbsfähigkeit der deutschen Milchwirtschaft, Münster-Hiltrup 2000, S. 44 f.

8 Vgl. Weindlmaier, H./Huber, A. (Fn. 5), S. 1087.

9 Bode, B.: Aktuelles Genossenschaftsrecht, Vortrag, gehalten auf dem 63. AgrarrechtSeminar, Goslar 2008, Folie 35.
} 
mehr geändert werden..$^{10}$ Deshalb zahlen Genossenschaftsmolkereien in der ersten Jahreshälfte meist weniger als die Nettoverwertung aus, gewähren aber in der zweiten Jahreshälfte oder am Jahresende eine Nachzahlung. ${ }^{11}$

Um die landwirtschaftlichen Lieferanten darüber hinaus zu entlohnen, nutzen Genossenschaftsmolkereien bereits heute unterschiedliche Instrumente. Beispiele hierfür sind Staffelprämien, die höhere Liefermengen belohnen sowie Bindungsprämien, die die langjährige Treue zum genossenschaftlichen Abnehmer honorieren. Diese sind vergleichbar mit den im genossenschaftlichen Bankenwesen seit 2009 vermehrt angebotenen Mitglieder-Bonus-Programmen. Diese Konzepte basieren auf der Intensität der Geschäftsbeziehung der einzelnen Mitglieder. Für bestimmte Transaktionen können Mitglieder Bonuspunkte sammeln, deren Gegenwert am Ende des Geschäftsjahres ausbezahlt wird. ${ }^{12}$

\section{b) Prinzipal-Agenten-Probleme der genossenschaftlichen Preisfindung}

Die eingetragene Genossenschaft hat den gesetzlich vorbestimmten Zweck der „Förderung des Erwerbs oder der Wirtschaft ihrer Mitglieder mittels gemeinschaft-

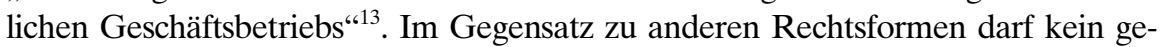
winnorientierter Zweck verfolgt werden. Genossenschaften sollten folglich die besten Konditionen für ihre Mitglieder anstreben. Dennoch müssen Preise derart gestaltet werden, dass Rücklagen gebildet und Investitionen durchgeführt werden können, um die dauerhafte Existenz zu sichern. ${ }^{14}$ Allerdings können Molkereien nur bei einer Unterschreitung der Nettoverwertung Rücklagen bilden und investieren. ${ }^{15}$

Der notwendige Umfang der Investitionen und Rücklagen ist für die Landwirte schwierig zu bewerten, da sie im Einzelfall entscheiden müssen, ob ihr Verzicht auf eine höhere Auszahlung zu einer sinnvollen strategischen Investition beiträgt. Diese Informationsasymmetrie führt zu Prinzipal-Agenten-Problemen. Eine empirische Studie zeigt, dass es auch Genossenschaften gibt, die unrentabel sind und trotzdem hohe Auszahlungspreise realisieren sowie solche, die gut wirtschaften und den Rohstoff schlecht entlohnen. ${ }^{16}$

$10 \quad$ Vgl. Bode, B. (Fn. 9), Folie 35.

11 Vgl. Weindlmaier, H./Huber, A. (Fn. 5), S. 1088.

12 Vgl. Jacobs, B.: Erlebbare Exklusivität. Genossenschaftliche Differenzierung per MitgliederBonus-Programm, URL: http://www.berndjacobs.de/BI_0209_S20.pdf, Abrufdatum: 15.02.2010; Neumann, M.: Mitglieder mit Bonus binden - Raiffeisenbank besinnt sich auf ihre Wurzeln, in: Taunuszeitung vom 06.08.2009.

13 Klose, H. (Fn. 2), S. 32.

14 Vgl. Klose, H. (Fn. 2), S. 32 f.

15 Vgl. Weindlmaier, H./Huber, A. (Fn. 5), S. 1087.

16 Vgl. Schlieckau, A./Paulmann, C./Theuvsen, L.: Jahresabschlussanalyse deutscher und österreichischer Molkereigenossenschaften, in: ZfgG 4/2008, S. 273 f. 
Für die Landwirte ist es also schwierig, in einem zunehmend internationalen und ausdifferenzierten Milchmarkt zu bewerten, ob ihre Genossenschaft eine falsche Unternehmensstrategie verfolgt und deshalb schlecht ausbezahlt oder ob bei niedrigen Auszahlungspreisen Prinzipal-Agenten-Probleme vorliegen, weil das Management vornehmlich Eigeninteressen (Gehälter, Boni, Karrierechancen) verfolgt. Diese Informationsasymmetrien treten nicht nur für das einfache Genossenschaftsmitglied, sondern aufgrund des Größenwachstums der Unternehmen auch für die Gremienmitglieder auf. In Kombination mit einem insgesamt niedrigen Auszahlungspreisniveau gerät das genossenschaftliche Preissystem infolgedessen unter Druck. Indizien für diese Situation sind der Milchstreik aus dem Jahr 2008 und die anhaltenden Proteste der Milchlandwirte, die auch Genossenschaftsmolkereien betreffen. Der starke Lieferantenverlust der Genossenschaftsmolkerei Nordmilch in der Vergangenheit ist ein weiteres Beispiel dafür. ${ }^{17}$

Dennoch erfüllt das derzeitige Preissystem im Gegensatz zu alternativen Preissystemen (Referenzpreis bzw. Verhandlungslösung) die Anforderungen der genossenschaftlichen Rechtsform in besonderer Weise und trägt dem Genossenschaftsgedanken Rechnung. Vor diesem Hintergrund soll das derzeitige Preissystem aus Sicht der Landwirte mit Hilfe eines verhaltenswissenschaftlichen Ansatzes untersucht werden.

\section{Entwicklung eines Modells zur Messung der Einflussfaktoren auf die Bewertung der genossenschaftlichen Preisfindung}

Der Milchstreik 2008 und die aktuelle Diskussion lassen vermuten, dass das derzeitige genossenschaftliche Preissystem in der Milchwirtschaft Unzufriedenheit hervorruft. Bisher gibt es aber kaum wissenschaftliche Untersuchungen zur Perspektive der Landwirte, deshalb soll diese mit Hilfe einer empirischen Untersuchung näher betrachtet werden.

Zur Analyse komplexer verhaltenswissenschaftlicher Fragestellungen finden insbesondere Kausalmodelle Anwendung. Die Hypothesen, die durch ein Kausalmodell abgebildet werden sollen, wurden im Vorfeld der empirischen Untersuchung auf Basis von theoretischen und praktisch-konzeptionellen Überlegungen abgeleitet und in Tabelle $1^{18}$ (S. 211) zusammengefasst. Im Folgenden werden die einzelnen Hypothesen kurz vorgestellt.

Die genossenschaftlichen Grundprinzipien Selbsthilfe, Selbstverwaltung und Selbstverantwortung sowie der genossenschaftliche Förderungsauftrag machen deutlich, dass die eingetragene Genossenschaft nicht nur ein Rechtsbegriff ist, sondern auch soziale und wirtschaftliche Gesichtspunkte umfasst. ${ }^{19}$ Da die genossen-

17 Vgl. o. V.: Molkereien streiten sich um Milch, in: Lebensmittelzeitung 59/2007, S. 16.

18 Eigene Darstellung.

19 Vgl. Turner, G.: Die eingetragene Genossenschaft (eG) im Vergleich der Gesell- 
Tab. 1: Zusammenfassung der Hypothesen

H1 Je positiver die Einstellung zur Rechtsform der Genossenschaft ist, desto besser wird das genossenschaftliche Preissystem bewertet.

H2 Mitglieder von Genossenschaften bewerten die genossenschaftliche Preisfindung besser als Nicht-Mitglieder.

H3a Je ausgeprägter die Langfristorientierung des Betriebsleiters ist, desto positiver ist die Einstellung zur Genossenschaft.

H3b Je ausgeprägter die Langfristorientierung des Betriebsleiters ist, desto besser wird das genossenschaftliche Preissystem bewertet.

H4 Je besser die Lieferantenorientierung der Molkerei ist, desto positiver ist die Bewertung der genossenschaftlichen Preisfindung.

H5 Ein hohes Preisvertrauen wirkt sich positiv auf die Bewertung des genossenschaftlichen Preissystems aus.

H6 Je mehr Macht dem Lebensmitteleinzelhandel zugesprochen wird, desto schlechter wird das genossenschaftliche Preissystem bewertet.

H7 Je größer der Betrieb ist, desto schlechter wird die genossenschaftliche Preisfindung bewertet.

Je höher die Milchleistung ist, desto schlechter wird das genossenschaftliche Preissystem bewertet.

Je höher der Spezialisierungsgrad (Einkommensanteil der Milcherzeugung am

H9 Gesamteinkommen) ist, desto schlechter wird die genossenschaftliche Preisfindung bewertet.

$\mathrm{H} 10$ Je älter die Befragten sind, desto besser wird die genossenschaftliche Preisfindung bewertet.

schaftliche Preisfindung auf den Genossenschaftsprinzipien aufbaut, wird davon ausgegangen, dass eine positive Einstellung zur Rechtsform der Genossenschaft die Bewertung der genossenschaftlichen Preisfindung positiv beeinflusst (H1). Es ist anzunehmen, dass Genossenschaftslieferanten das genossenschaftliche Preissystem positiver beurteilen als Lieferanten von Privatmolkereien, da sie über Vorstand und Aufsichtsrat Einfluss auf die Geschäftsführung nehmen können (H2) ${ }^{20}$

Landwirtschaftliche Betriebe sind meist seit Generationen in Familienbesitz, so

schaftsformen, in: Steding, R. (Hrsg.): Genossenschaftsrecht im Spannungsfeld von Bewahrung und Veränderung, Berliner Schriften zum Genossenschaftswesen Bd. 5 (1994), Göttingen, S. 23-25.

20 Vgl. Wocken, C./Spiller, A. (Fn. 5), S. 206. 
dass Familie und Unternehmen eine langfristige, generationenübergreifende Perspektive aufweisen. ${ }^{21}$ Die Bindung an Genossenschaftsmolkereien ist ebenfalls längerfristig orientiert. Die Mitgliedschaft besteht bis zur eigenen Kündigung, die meist durch eine Frist von zwei Jahren bestimmt ist. ${ }^{22}$ Die Genossenschaft ihrerseits kann dem Mitglied nur bei grobem Fehlverhalten kündigen. Es wird vermutet, dass eine hohe Langfristorientierung sowohl die Einstellung zur Genossenschaft als auch zu deren Preisfindung positiv beeinflusst ( $\mathrm{H} 3 \mathrm{a}$ und $\mathrm{H} 3 \mathrm{~b}$ ).

Die Lieferantenorientierung umfasst die Wahrnehmung von Zielkongruenz zwischen Landwirten und Molkereien, die empfundene Landwirtschaftsnähe und die Einflussmöglichkeiten der Landwirte. ${ }^{23}$ Eine hohe Lieferantenorientierung sollte zu einer besseren Bewertung der genossenschaftlichen Preisfindung führen (H4), da ein optimiertes Lieferantenmanagement unter anderem die Vorteile und Ziele des Preissystems kommuniziert.

Diller definiert Preisvertrauen als die Hoffnung oder Erwartung eines Lieferanten, dass der Geschäftspartner die Preisfindung nicht opportunistisch gestaltet. ${ }^{24}$ Wird die Preisgestaltung der Molkerei als nicht opportunistisch bewertet, kann das Preissystem auch in Zeiten niedriger Milchpreise als fair empfunden werden. Demnach verbessert ein hohes Preisvertrauen die Bewertung des genossenschaftlichen Preissystems (H5).

Die hohe Konzentration der Unternehmen im Lebensmitteleinzelhandel (LEH) führt zu Machtasymmetrien innerhalb der Wertschöpfungskette. Die vom Verwertungsergebnis abhängigen Erzeugerpreise werden vom LEH, der 40 \% der Milchprodukte abnimmt ${ }^{25}$ mitbestimmt. Dies kann von den Milcherzeugern als Schwäche des Preissystems interpretiert werden, so dass das Preissystem schlechter beurteilt wird, wenn die Macht des LEH - und damit der Einfluss des LEH auf den Milchpreis - als stark wahrgenommen wird (H6).

Als betriebsstrukturelle Merkmale gehen die Betriebsgröße, die Milchleistung und der Einkommensanteil der Milchproduktion am Gesamteinkommen in das Untersuchungsmodell ein (H7-H9). Größe und Milchleistung sind Indikatoren für die Leistungsfähigkeit eines Betriebes. Da leistungsfähigeren Betrieben eine höhere

${ }^{21}$ Vgl. Wimmer, R.: Erfolgsstrategien in Familie und Unternehmen, in: Frasl, E. J./Rieger, H. (Hrsg.): Family Business Handbuch, 1. Aufl., Wien 2007, S. 34 f.

22 Vgl. Wocken, C./Spiller, A.: Gestaltung von Milchlieferverträgen: Strategien für die Molkereiwirtschaft nach Auslaufen der Quote, in: Theuvsen, L./Schaper, C. (Hrsg.): Milchwirtschaft ohne Quote, 1. Aufl., Lohmar/Köln 2009, S. 117; Bode, B. (Fn. 9), Folie 41.

${ }^{23}$ Vgl. Wocken, C./Spiller, A. (Fn. 5), S. 212; Wocken, C./Spiller, A.: Sind hohe Milchauszahlungspreise wirklich alles? - Ergebnisse einer Studie zum Geschäftsbeziehungsmanagement, in: Theuvsen, L./Schaper, C. (Hrsg.): Milchwirtschaft ohne Quote, 1. Aufl., Lohmar/Köln 2009, S. 155.

${ }^{24}$ Vgl. Diller, H.: Preispolitik, 3. Aufl., Stuttgart 2000, S. 181.

25 Vgl. Zentrale Markt- und Preisberichtstelle für Erzeugnisse der Land-, Forst- und Ernährungswirtschaft GmbH: Wohin die Milch in Deutschland fließt, 2008, URL: http:// www.zmp.de/, Abrufdatum: 01.04.2009. 
Wechselbereitschaft unterstellt wird ${ }^{26}$ stehen sie Genossenschaften mit traditionell langfristigen Geschäftsbeziehungen eher kritisch gegenüber. Infolgedessen kann von einer negativeren Bewertung der Preisfindung ausgegangen werden. Als soziodemografische Variable wird der Einfluss des Alters betrachtet (H10).

\section{Datengrundlage und Methodik}

\section{a) Studiendesign}

Die explorative Studie soll einen ersten Eindruck von der Bewertung des genossenschaftlichen Preissystems aus Sicht der landwirtschaftlichen Lieferanten vermitteln. Von Mitte November bis Mitte Dezember 2008 wurden 161 milcherzeugende Landwirte mit einem ausführlichen, standardisierten Fragebogen persönlich interviewt. Der Schwerpunkt der Befragung lag auf großen Betrieben in Nordwestdeutschland. Die Stichprobe ist demnach nicht für die Gesamtheit der deutschen Milcherzeuger repräsentativ, lässt aber als aussagekräftige Sondierungsstudie Rückschlüsse auf große, zukunftsfähige Milchproduzenten zu.

Um die Einstellungen der Milcherzeuger zu erfassen, wurden siebenstufige LikertSkalen eingesetzt. Diese sind von -3 (lehne voll und ganz ab) über 0 (teils/teils) bis +3 (stimme voll und ganz zu) kodiert.

\section{b) Charakterisierung der Stichprobe}

Der Fokus der Studie liegt auf den Bundesländern Niedersachsen, Hessen und Schleswig-Holstein, in denen 75,7 \% der Befragungen durchgeführt wurden. In Tabelle $2^{27}$ sind die Durchschnittswerte der Stichprobe den Betriebsstrukturdaten dieser Bundesländer gegenüber gestellt.

Entsprechend der Auswahl der Betriebe ist der Anteil größerer Milchproduzenten in der Stichprobe höher als in der Grundgesamtheit. Die befragten Unternehmer halten durchschnittlich 95 Milchkühe und sind damit deutlich größer als der Durchschnittsbetrieb in Deutschland sowie in den Hauptbefragungsregionen. Auch die durchschnittlich bewirtschaftete Fläche liegt mit 221,7 ha wesentlich über dem Bundesmittel. Die Milchleistung, eine zentrale Kennziffer für die produktionswirtschaftliche Leistungsfähigkeit, liegt in der Stichprobe mit durchschnittlich $8.822 \mathrm{~kg}$ pro Kuh und Jahr ebenfalls deutlich über den deutschen Durchschnittswerten (vgl. Tabelle 2, S. 214).

\footnotetext{
26 Vgl. Wocken, C./Spiller, A. (Fn. 5), S. 13, 22 f.

27 Eigene Erhebung; Vgl. Statistisches Bundesamt: Statistisches Jahrbuch 2008, Wiesbaden 2008, S. 332, 361.
} 
Tab. 2: Betriebsstrukturen im Untersuchungsgebiet und in der Stichprobe

\begin{tabular}{llllll}
\hline & $\begin{array}{l}\text { Stich- } \\
\text { probe }\end{array}$ & Deutschland & $\begin{array}{l}\text { Nieder- } \\
\text { sachsen }\end{array}$ & Hessen & $\begin{array}{l}\text { Schleswig- } \\
\text { Holstein }\end{array}$ \\
\hline $\begin{array}{l}\text { Durchschnittliche } \\
\text { Betriebsgröße (ha) }\end{array}$ & 221,7 & 45,3 & 52,5 & 35,1 & 57,7 \\
\hline $\begin{array}{l}\text { Durchschnittliche Milchleistung } \\
\text { pro Kuh und Jahr (kg) }\end{array}$ & 8.822 & 6.944 & 7.080 & 6.734 & 7.014 \\
\hline $\begin{array}{l}\text { Durchschnittliche Anzahl } \\
\text { Milchkühe pro Betrieb }\end{array}$ & 95 & 40 & 50 & 34 & 62 \\
\hline
\end{tabular}

Der Anteil männlicher Befragter liegt bei 95,5\%. Mit knapp 39 Jahren ist das durchschnittliche Alter der befragten Milcherzeuger etwas niedriger als das Bundesmittel. ${ }^{28}$ Die Befragten sind Hauptentscheidungsträger der Betriebe: $66,5 \%$ der Probanden sind aktuell Betriebsleiter, bei 29,1\% handelt es sich um den Hofnachfolger. Der Ausbildungsstand in der Stichprobe ist hoch. 71,2 \% der Probanden liefern ihre Milch an eine Genossenschaftsmolkerei.

\section{Ergebnisse der empirischen Studie}

Das in Kapitel III entwickelte Modell wurde mittels des Partial-Least-Squares-Verfahrens (PLS) unter Verwendung des Programms SmartPLS Version 2.0 M3 geschätzt. ${ }^{29}$ Die PLS-Methode ist ein nicht-parametrisches Testverfahren, ${ }^{30}$ das gewählt wurde, da die Studie explorativ angelegt ist - bisher wurden keine vergleichbaren Untersuchungen durchgeführt - und die verwendete Datengrundlage auf einer relativ kleinen Stichprobe basiert. ${ }^{31}$

28 Vgl. Statistisches Bundesamt: Bevölkerung Deutschlands bis 2050 - 11. koordinierte Bevölkerungsvorausberechnung, Wiesbaden 2006, S. 17-19.

${ }_{29}$ Vgl. Ringle, C. M./Wende, S./Will, A.: SmartPLS 2.0 (beta), Universität Hamburg 2005; URL: http://smartpls.de, Abrufdatum: 27.04.2010.

30 Vgl. Ringle, C. M.: Gütemaße für den Partial Least Squares-Ansatz zur Bestimmung von Kausalmodellen, Arbeitspapier Nr. 16, Institut für Industriebetriebslehre und Organisation, Universität Hamburg 2004, S. 13.

${ }_{31}$ Vgl. Gefen, D./Straub, D./Boudreau, M.: Structural equation modelling and regression: Guidelines for research practice, in: Communications of the Association for Information Systems 4/2000, S. 6; Götz, O./Liehr-Gobbers, K.: Analyse von Strukturgleichungsmodellen mit Hilfe der Partial-Least-Squares (PLS)-Methode, in: Die Betriebswirtschaft 6/2004, S. 714. 


\section{a) Zur Bewertung des genossenschaftlichen Preissystems}

Wie in Kapitel II angedeutet, gibt es für Molkereien theoretisch verschiedene Möglichkeiten, den Milchpreis festzusetzen. 65,0 \% der Befragten bewerten die aktuelle genossenschaftliche Preisfindung kritisch. 35,0 \% finden diese Art der Milchpreisfindung gut oder stehen ihr indifferent gegenüber.

Alternative Preissysteme (Referenzpreise oder Preisverhandlungen) werden deutlich positiver wahrgenommen. Die negative Bewertung der genossenschaftlichen Preisfindung zeigt den Handlungsbedarf der Genossenschaftsmolkereien auf, wenn das gegenwärtige Preissystem Bestand haben soll (vgl. Tabelle $3^{32}$ ).

Tab. 3: Bewertung der verschiedenen Preissysteme

\begin{tabular}{lll}
\hline & $\mu^{1}$ & $\sigma^{2}$ \\
\hline Die Molkerei setzt die Milchpreise fest. $^{3}$ & $-0,86$ & 1,670 \\
Der Milchpreis wird von Erzeugergemeinschaften und der Molkerei $^{\text {ausgehandelt. }}{ }^{3}$ & 1,27 & 1,404 \\
Der Milchpreis wird in Bezug auf einen Referenzpreis festgelegt. $^{3}$ & 0,33 & 1,524 \\
\hline
\end{tabular}

${ }^{1}$ Mittelwert $;{ }^{2}$ Standardabweichung $;{ }^{3}$ Skala von +3 ,Finde ich sehr gut" bis -3 „,Finde ich sehr schlecht".

\section{b) Ergebnisse des reflektiven Messmodells}

Die Zusammenhänge zwischen den Konstrukten und den zugeordneten, beobachtbaren Items werden durch das Messmodell betrachtet (vgl. Tabelle $4^{33}$, S. 216). Durch Inhaltsvalidität, Indikatorreliabilität, Konstruktreliabilität und Diskriminanzvalidität wird die Güte des Messmodells geprüft. ${ }^{34}$ Alle Gütekriterien werden erfüllt, so dass von einem hinreichend verlässlichen und validen Messmodell ausgegangen werden kann. ${ }^{35}$

\section{c) Ergebnisse des Strukturmodells}

Das Strukturmodell dient der Überprüfung der auf Basis von theoretischen und praktisch-logischen Überlegungen hergeleiteten Zusammenhänge zwischen den Konstrukten.

\footnotetext{
32 Eigene Erhebung.

33 Eigene Erhebung.

34 Vgl. Götz, O./Liehr-Gobbers, K. (Fn. 31), S. 227 f.

35 Die Ergebnisse der Gütekriterien können bei den Autoren angefordert werden.
} 


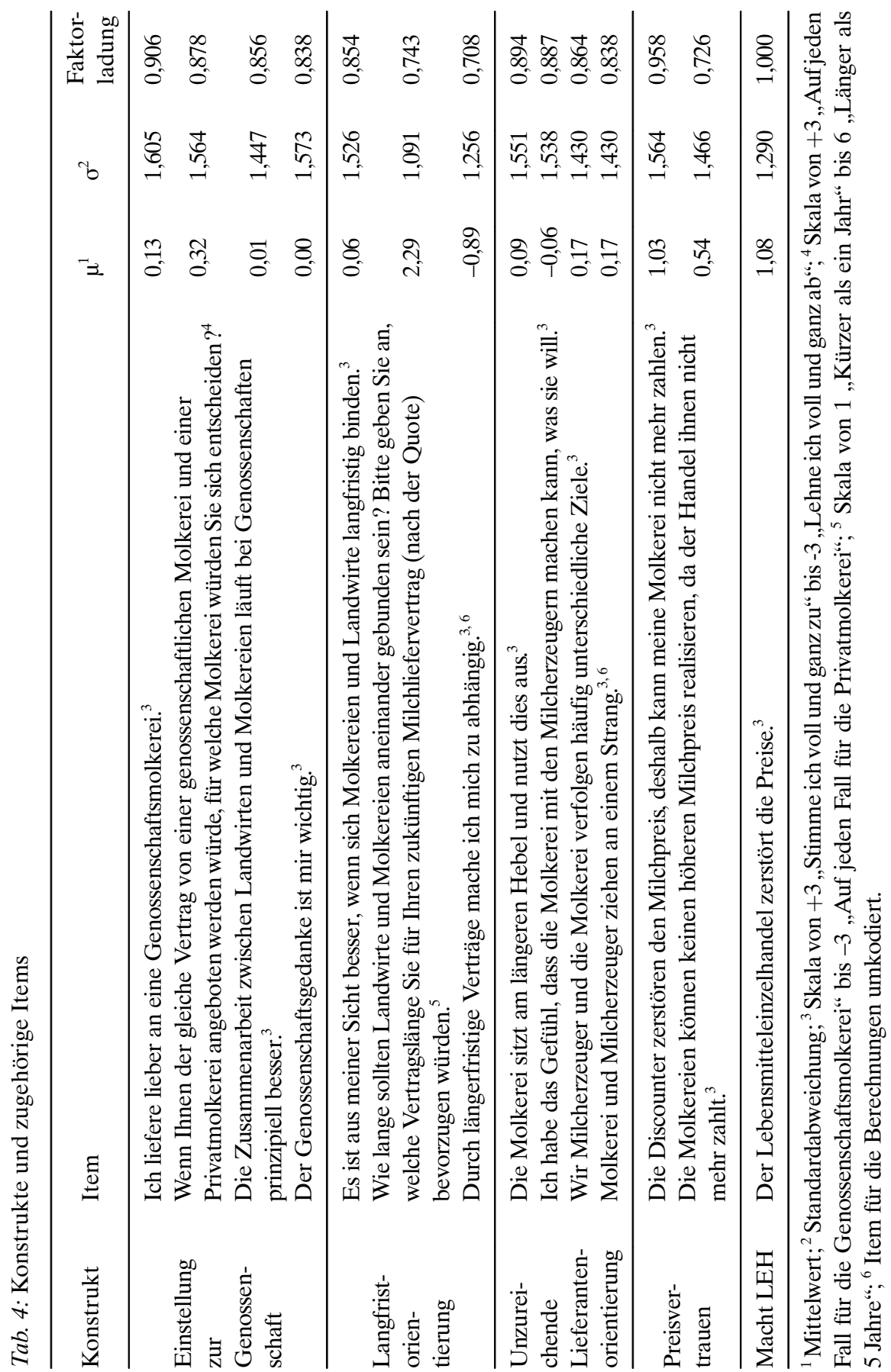

ZfgG 60,3, S. 206-220, ISSN 0044-2429

(C) Vandenhoeck \& Ruprecht GmbH \& Co. KG, Göttingen 2010 
Das Bestimmtheitsmaß $\left(R^{2}\right)$ ist ein globales Gütemaß zur Prüfung der Regression und gibt den Anteil der erklärten Streuung an der Gesamtstreuung eines Konstruktes an. Der Pfadkoeffizient gibt die Richtung (Vorzeichen) und Stärke (absoluter Wert) des Zusammenhangs zwischen zwei Konstrukten an. Die Signifikanz wird durch das Bootstrapping-Verfahren überprüft. ${ }^{36}$ Die Bestimmtheitsmaße sowie die Pfadkoeffizienten mit den Signifikanzniveaus sind in Abbildung $2^{37}$ dargestellt.

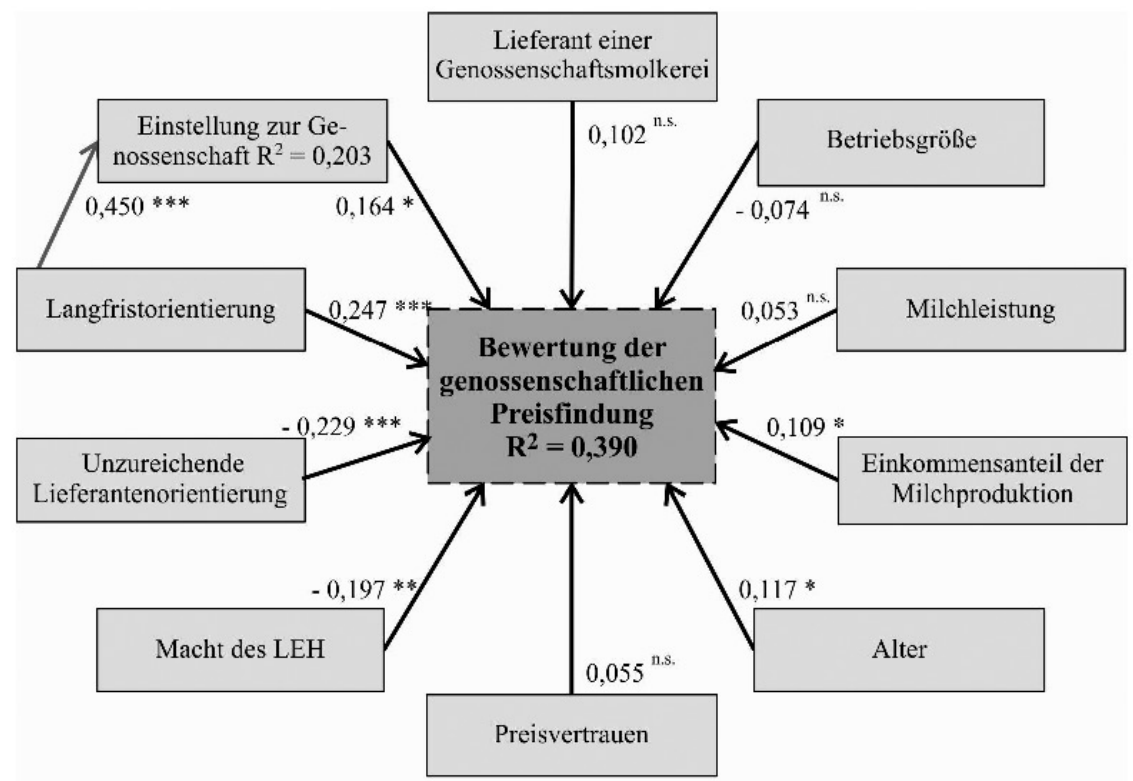

$A b b$. 2: Strukturmodell zur Erklärung der Bewertung der genossenschaftlichen Preisfindung ( ${ }^{\text {n.s. }}$ nicht signifikant, * Signifikanzniveau $5 \%$, ** Signifikanzniveau $1 \%$, *** Signifikanzniveau $0,1 \%$ )

Durch die in das Modell integrierten Konstrukte wird die Varianz der Bewertung der genossenschaftlichen Preisfindung zu 39,0 \% $\left(\mathrm{R}^{2}=0,390\right)$ erklärt.

Das Konstrukt Langfristorientierung hat den höchsten Einfluss vor einer unzureichenden Lieferantenorientierung. Damit können die Hypothesen H3b und H4 bestätigt werden. Der nächstbedeutende Einflussfaktor ist die Macht des LEH. Hypothese H6, die besagt, dass das genossenschaftliche Preissystem schlechter bewertet wird, wenn dem LEH viel Macht zugesprochen wird, wird damit unterstützt. Der

36 Vgl. Jahn, S.: Strukturgleichungsmodellierung mit LISREL, AMOS und SmartPLS, 1. Aufl., Chemnitz 2007, S. 9 f., 18 f.; Ringle, C. M. (Fn. 30), S. 13, 18.

37 Eigene Darstellung und Erhebung. 
Einfluss des Konstruktes Einstellung zur Genossenschaft ist signifikant, somit kann H1 bestätigt werden.

Der Einkommensanteil der Milchproduktion und das Alter haben einen signifikanten, aber geringen Einfluss auf die Bewertung des genossenschaftlichen Preissystems (H9 und H10). Aufgrund des nicht signifikanten Einflusses der Konstrukte Lieferant einer Genossenschaft, Preisvertrauen, Betriebsgröße und Milchleistung müssen die Hypothesen $\mathrm{H} 2, \mathrm{H} 5, \mathrm{H} 7$ und $\mathrm{H} 8$ abgelehnt werden.

Die Varianz des Konstruktes Einstellung zur Genossenschaft kann durch das Konstrukt Langfristorientierung zu 20,3 \% $\left(\mathrm{R}^{2}=0,203\right)$ erklärt werden. Der Pfadkoeffizient beträgt 0,450 und zeigt einen positiven Einfluss, was H3a stützt.

\section{Diskussion der Ergebnisse}

In der theoretischen Analyse wurde das bestehende Preissystem als prinzipiell geeignetes System für genossenschaftlich organisierte Molkereiunternehmen bewertet, denn mit diesem wird vor allem einer langfristigen Existenzsicherung sowie dem Genossenschaftsgedanken Rechnung getragen. Es kann allerdings festgehalten werden, dass die genossenschaftliche Preisfindung von den befragten Landwirten am negativsten bewertet wird. Die Möglichkeit von Preisverhandlungen zwischen Molkerei und Milcherzeuger wird derzeit am stärksten präferiert. Dies verwundert insofern, als ein solches System die gesetzlich feststehenden Einflussstrukturen der Anteilseigner unterlaufen würde. Dennoch zeigt die schlechte Bewertung der genossenschaftlichen Preisfindung den Handlungsbedarf der Genossenschaftsmolkereien auf.

Mit Hilfe eines Kausalmodells wurden verschiedene Einflussfaktoren auf die Bewertung der genossenschaftlichen Preisfindung identifiziert. Diese liegen allerdings nur teilweise im Einflussbereich der Molkerei.

Auf die Langfristorientierung der Lieferanten, die unter anderem durch die generationenübergreifende Perspektive der landwirtschaftlichen Familienbetriebe geprägt ist ${ }^{38}$ kann die Molkerei nicht direkt einwirken. Allerdings hat diese einen positiven Einfluss auf die Bewertung der genossenschaftlichen Preisfindung und auf die Einstellung zur Genossenschaft, die wirtschaftliche und soziale Aspekte der Rechtsform Genossenschaft berücksichtig ${ }^{39}$ und ebenfalls die Bewertung der Preisfindung verbessert. Der Einfluss der Einstellung zur Genossenschaft sowie der Einfluss der tatsächlichen Mitgliedschaft in einer Genossenschaft sind jedoch geringer als erwartet.

Einen weiteren bestimmenden Faktor stellt die Lieferantenorientierung der Molkerei dar, die das Vorhandensein gemeinsamer Ziele, die empfundene Landwirtschaftsnähe und den Einfluss der Landwirte auf die Unternehmenspolitik um-

\footnotetext{
38 Vgl. Wimmer, R. (Fn. 21), S. 34 f.

39 Vgl. Turner, G. (Fn. 19), S. 23-25.
} 
fasst. Eine Optimierung der Lieferantenorientierung kann den Genossenschaftsmolkereien zum Beispiel durch die Reaktivierung dessen, was in der Literatur häufig als „Genossenschaftsgeist“ beschrieben wird, gelingen. ${ }^{40}$ Infolgedessen kann die Bewertung des Preissystems deutlich verbessert werden. Möglicherweise können die in Kapitel II a) diskutierten Mitglieder-Bonus-Programme einen Beitrag zu einer stärkeren Mitgliederbindung leisten. Dies kann indirekt eine Verbesserung der Bewertung des Genossenschaftssystems bewirken. Eine positive Einstellung zur Genossenschaft kann, wie bereits illustriert, eine vorteilhafte Einschätzung der genossenschaftlichen Preisfindung nach sich ziehen.

Nicht von den Molkereien beeinflussbar sind die betriebsstrukturellen und soziodemografischen Einflussgrößen. Ein signifikant positiver Einfluss geht vom Einkommensanteil der Milchproduktion am Gesamteinkommen aus. Dieser lässt auf den Spezialisierungsgrad der Betriebe und damit auf das Interesse an der Preisfindung schließen. Auch ein hohes Alter der Befragten wirkt sich positiv auf die Bewertung aus. Folglich besteht gerade bei jungen Lieferanten die Notwendigkeit, das bestehende System der Preisfindung zu verbessern und anders zu kommunizieren.

Es wurde kein statistisch signifikanter Einfluss des Preisvertrauens auf die Bewertung der genossenschaftlichen Preisfindung gemessen. Eine hohe wahrgenommene Macht des LEH beeinflusst die Bewertung der Preisfindung hingegen negativ. Landwirte sehen im LEH häufig den dominierenden Akteur in der Wertschöpfungskette. Das genossenschaftliche System der Preisfindung, bei dem sie den Preis rückwirkend mitgeteilt bekommen, ist in einer solchen Situation aus ihrer Sicht dann möglicherweise zu reaktiv. Wie der Milchstreik zeigte, wollen sie selbst gerne mehr Druck ausüben.

Die negative Bewertung der genossenschaftlichen Preisfindung lässt deshalb auf Reaktanz schließen. Durch die einseitige Festsetzung des monatlichen Erzeugerpreises sieht der Landwirt seine Entscheidungsfreiheit bedroht und reagiert unter anderem mit Widerstand (Ablehnung des Preissystems) sowie dem Schaffen einer Alternative (z. B. Gründung des Bundesverbands deutscher Milchviehhalter e. V.). ${ }^{41}$

\section{Handlungsempfehlungen}

Um Reaktanz abzubauen, müssen die Genossenschaftsmolkereien die Lieferantenorientierung optimieren und das Preissystem möglichst transparent und nachvollziehbar gestalten. Die Molkerei kann Verbesserungen schaffen, indem gemeinsame Ziele sowie ein partnerschaftlicher und fairer Umgang mit den Lieferanten in den

40 Vgl. Österberg, P./Nilsson, J.: Members' Perception of Their Participation in the Governance of Cooperatives. The Key to Trust and Commitment in Agricultural Cooperatives, in: Agribusiness vol. 25 (2009), S. 186 f.

${ }^{41}$ Vgl. Gniech, G./Dickenberger, D.: Die Reaktanz-Theorie, in: Kieselbach, T. (Hrsg.): Bremer Beiträge zur Psychologie, Bremen 1992, S. 6-9. 
Mittelpunkt des Lieferantenmanagements gerückt werden ${ }^{42}$ um den „Genossenschaftsgeist" neu zu aktivieren.

Die Molkerei kann weiter über die Langfristorientierung und die Einstellung zur Genossenschaft auf die Bewertung des Preissystems einwirken, indem einerseits die langfristige strategische Ausrichtung der Preisfindung und andererseits die Werte der Genossenschaft, in Zeiten einer wachsenden Konzentration auf den nachfolgenden Wertschöpfungsstufen, kommuniziert und in den Blickwinkel gerückt werden. Dafür sollten die vorhandenen Instrumente der Mitgliederbindung (z. B. Staffelprämie, Bindungsprämie) vermehrt genutzt werden.

Ferner sollte die als bedroht wahrgenommene Entscheidungsfreiheit der Landwirte wiederhergestellt werden. Dies kann zum Beispiel durch das Schaffen einer Wahlalternative erfolgen. Den Landwirten kann beispielsweise die Wahl zwischen dem klassischen genossenschaftlichen Preissystem und einem Mehr-Monats-Gleitpreis eröffnet werden. Dieser Gleitpreis sollte auf einem für mehrere Monate garantierten Mindestpreis basieren, um den Landwirten etwas mehr Planungssicherheit zu gewährleisten. Auch könnten bessere Preisprognosedaten und eine aktivere Kommunikation des landwirtschaftlichen Einflusses auf die Preisfindung das Gefühl des Ausgeliefertseins abbauen.

Eine Verbesserung der Verhandlungsmacht gegenüber dem LEH scheint sinnvoll. Die aktuelle Vertriebskooperation der beiden größten deutschen genossenschaftlichen Molkereien illustriert entsprechende Ansätze im Molkereisektor. ${ }^{43}$

Die vorliegende Studie deckt einige Handlungsfelder auf, die es Genossenschaftsmolkereien ermöglichen, die Bewertung ihres klassischen Preissystems positiv zu beeinflussen, um trotz veränderter Rahmenbedingungen im Wettbewerb bestehen zu können. Abschließend wird auf den explorativen Charakter der Studie verwiesen, der in der innovativen Fragestellung begründet liegt. Es besteht zudem weiterer Forschungsbedarf, inwieweit die analysierten Determinanten auf andere Branchen der Agrarwirtschaft (Zucker-, Wein-, Obst- und Gemüsewirtschaft) übertragen werden können. Angesichts der hohen Relevanz genossenschaftlicher Verarbeitungsunternehmen im gesamten Agribusiness stellt die Zufriedenheit der Genossenschaftsmitglieder mit dem Auszahlungspreissystem einen wichtigen und in der Forschung bisher unbeachteten Faktor für die Zukunft der Rechtsform dar.

\footnotetext{
$42 \quad$ Vgl. Wocken, C./Spiller, A. (Fn. 5), S. 22-24.

43 Vgl. LEBENSMITTELZEITUNG NET: Humana und Nordmilch weiten Zusammenarbeit aus, URL: http://www.lz-net.de/, Abrufdatum: 01.04.2009.
} 